\title{
ARTICLES
}

\section{Broadcasting of entanglement via local copying}

\author{
V. Bužek, ${ }^{1,2}$ V. Vedral, ${ }^{1}$ M. B. Plenio, ${ }^{1}$ P. L. Knight, ${ }^{1}$ and M. Hillery ${ }^{3}$ \\ ${ }^{1}$ Optics Section, The Blackett Laboratory, Imperial College, London SW7 2BZ, England \\ ${ }^{2}$ Institute of Physics, Slovak Academy of Sciences, Dubravská cesta 9, 84228 Bratislava, Slovakia \\ ${ }^{3}$ Department of Physics and Astronomy, Hunter College, City University of New York, 695 Park Avenue, New York, New York 10021
}

(Received 13 November 1996)

\begin{abstract}
We show that inseparability of quantum states can be partially broadcasted (copied or cloned) with the help of local operations, i.e., distant parties sharing an entangled pair of spin-1/2 states can generate two pairs of partially nonlocally entangled states using only local operations. This procedure can be viewed as an inversion of quantum purification procedures. [S1050-2947(97)02905-3]

PACS number(s): 03.65.Bz
\end{abstract}

\section{INTRODUCTION}

The laws of quantum mechanics impose restrictions on manipulations with quantum information. These restrictions can, on the one hand, be fruitfully utilized in quantum cryptography [1]. On the other hand, they put limits on the precision with which quantum-mechanical measurements or copying (broadcasting and cloning) of quantum information can be performed [2-4]. One of the most important aspects of quantum-information processing is that information can be "encoded" in nonlocal correlations (entanglement) between two separated particles. The more "pure" the quantum entanglement, the more "valuable" the given twoparticle state. This explains current interest in purification procedures [5] by means of which one can extract pure quantum entanglement from a partially entangled state. In other words, it is possible to compress locally an amount of quantum information. This is implemented as follows. Two "distant" parties share a number of partially entangled pairs. They each then apply local operations on their own particles and, depending on the outcomes (which they are allowed to communicate classically), they agree on further actions. By doing this they are able to reduce the initial ensemble to a smaller one but whose pairs are more entangled. This has important implications in the field of quantum cryptography as it immediately implies an unconditional security of communication at the quantum level.

Our main motivation for the present work comes from the fact that local compression of quantum correlations is possible. We now ask the opposite: Can quantum correlations be "decompressed"? Namely, can two parties acting locally start with a number of highly entangled pairs and end up with a greater number of pairs with lower entanglement? This, if possible, would also be of great operational value in determining the amount of entanglement of a certain state [6]. For if we could optimally "split" the original entanglement of a single pair into two pairs equally entangled (e.g., having the same state) we have a means of defining half the entanglement of the original pair.

We may view the process of decompression of quantum entanglement (i.e., inseparability) as a local copying (broadcasting and cloning) of nonlocal quantum correlations. In this case one might raise the question whether it is possible to clone partially quantum entanglement using only local operations. When we ask the question whether inseparability can be broadcast via local copying we mean the following. Let two distant parties share an inseparable state $\hat{\rho}_{a_{I} a_{I I}}^{(i d d)}$. Now manipulate the two systems $a_{I}$ and $a_{I I}$ locally, e.g., with the help of two distant quantum copiers $X_{I}$ and $X_{I I}$. These two quantum copiers are supposed to be initially uncorrelated (or, more generally, they can be classically correlated, i.e., the density operator $\hat{\rho}_{x_{I} x_{I I}}$ describing the input state of two quantum copiers is separable). The quantum copier $X_{I}\left(X_{I I}\right)$ copies the quantum subsystem $a_{I}\left(a_{I I}\right)$ such that at the output two systems $a_{I}$ and $b_{I}\left(a_{I I}\right.$ and $\left.b_{I I}\right)$ are produced (see Fig. 1 ). As a result of this copying we obtain out of two systems $a_{I}$ and $a_{I I}$ four systems described by a density operator

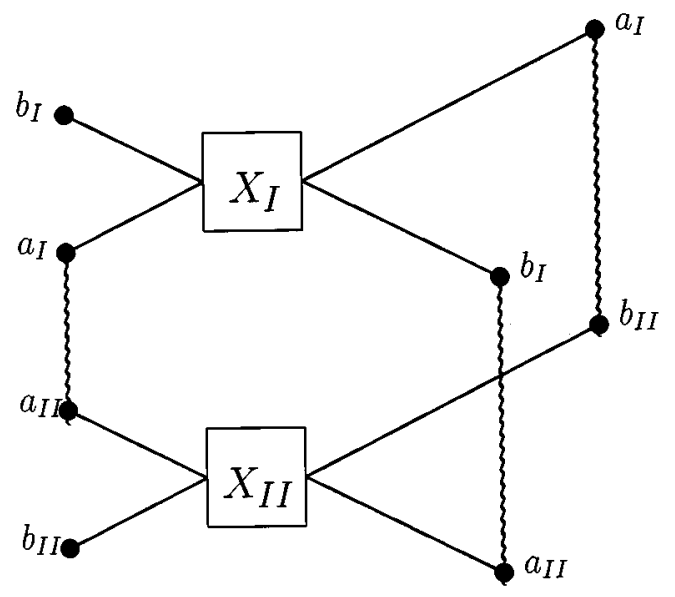

FIG. 1. An entangled pair of spin-1/2 particles $a_{I}, a_{I I}$ is shared by two distant parties $I$ and $I I$, which then perform local operations using two quantum copiers $X_{1}$ and $X_{2}$. Each party obtains two output particles that are in a separable state, while the spatially separated pairs $a_{I}, b_{I I}$ and $a_{I I}, b_{I}$ are entangled. 
$\hat{\rho}_{a_{I} b_{I} a_{I I} b_{I I}}^{(\text {out }}$. If the states $\hat{\boldsymbol{\rho}}_{a_{I} b_{I I}(\text { out })}$ and $\hat{\boldsymbol{\rho}}_{a_{I I} b_{I}}^{(\text {out })}$ are inseparable while

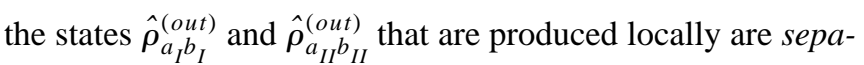
rable, then we say that we have partially broadcasted (cloned and split) the entanglement (inseparability) that was present in the input state. As we said earlier, this broadcasting of inseparability can be viewed as an inversion of the distillation protocol. The advantage of our operational definition is that we impose the inseparability condition only between two spins $1 / 2$ (i.e., either on spins $a_{I}$ and $b_{I I}$ or spins $a_{I I}$ and $\left.b_{I}\right)$. Obviously, due to the quantum nature of copying employed in our scheme, multiparticle quantum correlations between pairs of spins $a_{I} b_{I I}$ and $a_{I I} b_{I}$ (i.e., each of these systems is described in four-dimensional Hilbert space) may appear at the output. But presently there do not exist strict criteria that would allow one to specify whether these systems are inseparable (see below) and, consequently, it would be impossible to introduce operational definition of the inverse of the distillation protocol based on multiparticle inseparability.

In this paper we show that the decompression of initial quantum entanglement is indeed possible, i.e., that from a pair of entangled particles we can, by local operations, obtain two less entangled pairs. Therefore, entanglement can be copied locally, i.e., the inseparability can be partially broadcast.

\section{INSEPARABILITY AND THE PERES-HORODECKI THEOREM}

We first recall that a density operator of two subsystems is inseparable if it cannot be written as the convex sum

$$
\hat{\rho}_{a_{I} a_{I I}}=\sum_{m} w^{(m)} \hat{\boldsymbol{\rho}}_{a_{I}}^{(m)} \otimes \hat{\boldsymbol{\rho}}_{a_{I I}(m)} .
$$

Inseparability is one of the most fundamental quantum phenomena, which, in particular, may result in the violation of Bell's inequality (to be specific, a separable system always satisfies Bell's inequality, but the contrary is not necessarily true). Note that distant parties cannot prepare an inseparable state from a separable state if they only use local operations and classical communications.

We will not address the question of copying entanglement in its most general form, but will rather focus our attention on copying of the entanglement of spin-1/2 systems. In this case, we can explicitly describe the transformations that are necessary to broadcast entanglement. Moreover, in the case of two spins $1 / 2$ we can effectively utilize the PeresHorodecki theorem $[7,8]$, which states that the positivity of the partial transposition of a state is necessary and sufficient for its separability. Before we proceed further we briefly described how to "use"' this theorem: The density matrix associated with the density operator of two spins $1 / 2$ can be written as

$$
\rho_{m \mu, n \nu}=\left\langle e_{m}\left|\left\langle f_{\mu}|\hat{\rho}| e_{n}\right\rangle\right| f_{\nu}\right\rangle
$$

where $\left\{\left|e_{m}\right\rangle\right\}\left(\left\{\left|f_{\mu}\right\rangle\right\}\right)$ denotes an orthonormal basis in the Hilbert space of the first (second) spin 1/2 (for instance, $\left|e_{0}\right\rangle=|0\rangle_{a},\left|e_{1}\right\rangle=|1\rangle_{a}$ and $\left.\left|f_{0}\right\rangle=|0\rangle_{b},\left|f_{1}\right\rangle=|1\rangle_{b}\right)$. The partial transposition $\hat{\rho}^{T_{2}}$ of $\hat{\rho}$ is defined as

$$
\rho_{m \mu, n \nu}^{T_{2}}=\rho_{m \nu, n \mu} .
$$

Then the necessary and sufficient condition for the state $\hat{\rho}$ of two spins $1 / 2$ to be inseparable is that at least one of the eigenvalues of the partially transposed operator (3) is negative. This is equivalent to the condition that at least one of the two determinants

$$
\begin{gathered}
W_{3}=\operatorname{det}\left(\begin{array}{ccc}
\rho_{00,00}^{T_{2}} & \rho_{00,01}^{T_{2}} & \rho_{00,10}^{T_{2}} \\
\rho_{01,00}^{T_{2}} & \rho_{01,01}^{T_{2}} & \rho_{01,10}^{T_{2}} \\
\rho_{10,00}^{T_{2}} & \rho_{10,01}^{T_{2}} & \rho_{10,10}^{T_{2}}
\end{array}\right) \\
W_{4}=\operatorname{det}\left\{\rho^{T_{2}}\right\}
\end{gathered}
$$

is negative. In principle, one would also have to check the positivity of the subdeterminants $W_{1}=\rho_{00,00}^{T_{2}}$ and $W_{2}=\rho_{00,00}^{T_{2}} \rho_{01,01}^{T_{2}}-\rho_{00,01}^{T_{2}} \rho_{01,00}^{T_{2}}$. However, they are positive because the density operator $\hat{\rho}$ is positive. In this paper we deal exclusively with nonsingular operators $\rho^{T_{2}}$. Consequently, we do not face any problem that may arise when $\rho^{T_{2}}$ are singular.

\section{QUANTUM COPYING AND THE NO-BROADCASTING THEOREM}

In the realm of quantum physics there does not exist a process that would allow us to copy (clone and broadcast) an arbitrary state with perfect accuracy [2-4]. What this means is that if the original system is prepared in an arbitrary state $\hat{\rho}_{a}^{(i d)}$, then it is impossible to design a transformation

$$
\hat{\rho}_{a}^{(i d)} \rightarrow \hat{\rho}_{a b}^{(o u t)}
$$

where $\hat{\rho}_{a b}^{(o u t)}$ is the density operator of the combined originalcopy quantum system after copying such that

$$
\operatorname{Tr}_{b} \hat{\boldsymbol{\rho}}_{a b}^{(\text {out })}=\hat{\boldsymbol{\rho}}_{a}^{(i d)}, \quad \operatorname{Tr}_{a} \hat{\boldsymbol{\rho}}_{a b}^{(\text {out })}=\hat{\boldsymbol{\rho}}_{b}^{(i d)} .
$$

This is the content of the no-broadcasting theorem, which has been recently proven by Barnum et al. [3]. The stronger form of broadcasting, when

$$
\hat{\boldsymbol{\rho}}_{a b}^{(o u t)}=\hat{\boldsymbol{\rho}}_{a}^{(i d)} \otimes \hat{\boldsymbol{\rho}}_{b}^{(i d)},
$$

is denoted as the cloning of quantum states. Wootters and Zurek [2] pointed out that the cloning of an arbitrary pure state is impossible. To be more specific, the no-broadcasting and no-cloning theorems allow us to copy a single a priori known state with absolute accuracy. In fact, also two states can be precisely copied if it is a priori known that they are orthogonal. But if no a priori information about the copied (i.e., original) state is known, then precise copying (broadcasting) is impossible.

Even though ideal copying is prohibited by the laws of quantum mechanics, it is still possible to imagine quantum copiers that produce reasonably good copies without destroy- 
ing the original states too much. To be specific, instead of imposing unrealistic constraints on outputs of quantum copiers given by Eqs. (7) and (8), one can adopt a more modest approach and give an operational definition of a quantum copier. For instance, a reasonable quantum copier can be specified by three conditions.

(i) States of the original system and its quantum copy at the output of the quantum copier, described by density operators $\hat{\rho}_{a}^{(\text {out })}$ and $\hat{\rho}_{b}^{(\text {out })}$, respectively, are identical, i.e.,

$$
\hat{\rho}_{a}^{(\text {out })}=\hat{\rho}_{b}^{(\text {out })} .
$$

(ii) Once no a priori information about the in state of the original system is available, then it is reasonable to assume that all pure states are copied equally well. One way to implement this assumption is to design a quantum copier such that distances between density operators of each system at the output $\left(\hat{\rho}_{j}^{\text {(out) }}\right.$, where $\left.j=a, b\right)$ and the ideal density operator $\hat{\rho}^{(i d)}$, which describes the in state of the original mode, are input-state independent. Quantitatively, this means that if we employ the Bures distance [9]

$$
d_{B}\left(\hat{\rho}_{1} ; \hat{\rho}_{2}\right):=\sqrt{2}\left[1-\operatorname{Tr}\left(\hat{\rho}_{1}^{1 / 2} \hat{\rho}_{2} \hat{\rho}_{1}^{1 / 2}\right)^{1 / 2}\right]^{1 / 2}
$$

as a measure of distance between two operators, then the quantum copier should be such that

$$
d_{B}\left(\hat{\rho}_{i}^{(\text {out })} ; \hat{\rho}_{i}^{(i d)}\right)=\mathrm{const}, \quad i=a, b .
$$

(iii) It is important to note that the copiers we have in mind are quantum devices. This means that even though we assume that a quantum copier is initially disentangled (let us assume it is in a pure state) from the input system it is most likely that after copying has been performed the copier will become entangled with the output original plus copy system. This entanglement is in part responsible for an irreversible noise introduced into the output original plus copy system). Consequently, $\hat{\boldsymbol{\rho}}_{a b}^{(\text {out })} \neq \hat{\boldsymbol{\rho}}_{a b}^{(i d)}$, where $\hat{\boldsymbol{\rho}}_{a b}^{(i d)}=\hat{\boldsymbol{\rho}}_{a}^{(i d)} \otimes \hat{\boldsymbol{\rho}}_{b}^{(i d)}$. Once again, if no a priori information about the state $\hat{\rho}_{a}^{(i d)}$ of the input system is known, it is desirable to assume that the copier is such that the Bures distance between the actual output state $\hat{\rho}_{a b}^{(\text {out })}$ of the original plus copy system and the ideal output state $\hat{\rho}_{a b}^{(i d)}$ is input-state independent, i.e.,

$$
d_{B}\left(\hat{\rho}_{a b}^{(o u t)} ; \hat{\rho}_{a b}^{(i d)}\right)=\text { const. }
$$

The copying process as specified by conditions (i)-(iii) can be understood as broadcasting in a weak sense, i.e., it is not perfect, but it can serve to some purpose when it is desirable to copy (at least partially) quantum information without destroying it completely (eavesdropping is one of the examples [10]).

The action of the quantum copier for spins $1 / 2$ that satisfies conditions (i)-(iii) can be described in terms of a unitary transformation of two basis vectors $|0\rangle_{a}$ and $|1\rangle_{a}$ of the original system. This transformation can be represented as [4]

$$
|0\rangle_{a}|\xi\rangle_{b}|Q\rangle_{x} \rightarrow \sqrt{\frac{2}{3}}|00\rangle_{a b}|\uparrow\rangle_{x}+\sqrt{\frac{1}{3}}|+\rangle_{a b}|\downarrow\rangle_{x},
$$

$$
|1\rangle_{a}|\xi\rangle_{b}|Q\rangle_{x} \rightarrow \sqrt{\frac{2}{3}}|11\rangle_{a b}|\downarrow\rangle_{x}+\sqrt{\frac{1}{3}}|+\rangle_{a b}|\uparrow\rangle_{x},
$$

where $|Q\rangle_{x}$ describes the initial state of the quantum copier, $|\xi\rangle_{b}$ is an arbitrary initial state of mode $b$, and $|\uparrow\rangle_{x}$ and $|\downarrow\rangle_{x}$ are two orthonormal vectors in the Hilbert space of the quantum copier. In Eq. (13) we use the notation such that $\left|e_{m} e_{n}\right\rangle_{a b}=\left|e_{m}\right\rangle_{a} \otimes\left|e_{n}\right\rangle_{b}$ and $|+\rangle_{a b}=\left(|01\rangle_{a b}+|10\rangle_{a b}\right) / \sqrt{2}$. We do not specify the in state of mode $b$ in Eq. (13). In our discussion there is no need to specify this state. Obviously, in real physical processes the in state of mode $b$ may play an important role. In what follows, unless it may cause confusion, we will omit subscripts indicating the subsystems.

\section{BROADCASTING OF INSEPARABILITY}

Now we present the basic operation necessary to copy entanglement locally for spins $1 / 2$. The scenario is as follows. Two parties $X_{I}$ and $X_{I I}$ share a pair of particles prepared in a state

$$
|\Psi\rangle_{a_{I} a_{I I}}=\alpha|00\rangle_{a_{I} a_{I I}}+\beta|11\rangle_{a_{I} a_{I I}}
$$

where we assume $\alpha$ and $\beta$ to be real and $\alpha^{2}+\beta^{2}=1$. The state (14) is inseparable for all values of $\alpha^{2}$ such that $0<\alpha^{2}<1$ because one of the two determinants $W_{j}$ from Eqs. (4) and (5) is negative. Now we assume that the system $a_{I}$ $\left(a_{I I}\right)$ is locally copied by the quantum copier $X_{I}\left(X_{I I}\right)$ operating according to the transformations (13). As the result of the copying we obtain a composite system of four spins $1 / 2$ described by the density operator $\hat{\rho}_{a_{I} b_{I} a_{I I} b_{I I} \text {.out }}$. We are now interested in seeing two properties of this output state. First, both states $\hat{\rho}_{a_{I} b_{I I}}^{(\text {out })}$ and $\hat{\rho}_{a_{I I} b_{I}}^{(\text {out })}$ should be inseparable simultaneously for at least some values of $\alpha$ and second, states $\hat{\rho}_{a_{I} b_{I}}^{(o u t)}$ and $\hat{\rho}_{a_{I I} b_{I I}}^{(o u t)}$ should be separable simultaneously for some values of $\alpha$ for which $\hat{\rho}_{a_{I} b_{I I}}^{\text {(out) }}$ and $\hat{\rho}_{a_{I I} b_{I}}^{\text {out })}$ are inseparable.

Using the transformation (13) we find the local output of the quantum copier $X_{I}$ to be described by the density operator

$$
\hat{\rho}_{a_{I} b_{I}}^{(\text {out })}=\frac{2 \alpha^{2}}{3}|00\rangle\left\langle 00\left|+\frac{1}{3}\right|+\right\rangle\left\langle+\left|+\frac{2 \beta^{2}}{3}\right| 11\right\rangle\langle 11|,
$$

while the nonlocal pair of output particles is in the state described by the density operator

$$
\begin{aligned}
\hat{\rho}_{a_{I^{b}} b_{I I}}^{(\text {out })=} & \frac{24 \alpha^{2}+1}{36}|00\rangle\left\langle 00\left|+\frac{24 \beta^{2}+1}{36}\right| 11\right\rangle\langle 11| \\
& +\frac{5}{36}(|01\rangle\langle 01|+| 10\rangle\langle 10|) \\
& +\frac{4 \alpha \beta}{9}(|00\rangle\langle 11|+| 11\rangle\langle 00|) .
\end{aligned}
$$

We note that due to the symmetry between the systems $I$ and

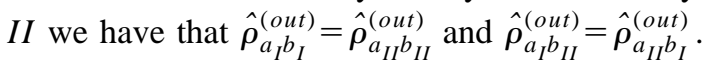

Now we check for which values of $\alpha$ the density operator $\hat{\rho}_{a_{I} b_{I I}}^{(o u t)}$ is inseparable. From the determinants in Eqs. (4) and 
(5) associated with this density operator it immediately follows that $\hat{\rho}_{a_{I} b_{I I}}^{(\text {out })}$ is inseparable if

$$
\frac{1}{2}-\frac{\sqrt{39}}{16} \leqslant \alpha^{2} \leqslant \frac{1}{2}+\frac{\sqrt{39}}{16} .
$$

On the other hand, from Eq. (15) we find that $\hat{\rho}_{a_{I} b_{I}}^{(\text {out })}$ is separable if

$$
\frac{1}{2}-\frac{\sqrt{48}}{16} \leqslant \alpha^{2} \leqslant \frac{1}{2}+\frac{\sqrt{48}}{16} .
$$

Comparing Eqs. (17) and (18) we observe that $\hat{\rho}_{a_{I} b_{I}}^{(\text {out })}$ is separable if $\hat{\rho}_{a_{I} b_{I I}}^{(\text {out }}$ is inseparable. This finally proves that it is possible to clone partially quantum entanglement using only local operations and classical communication. Note that any other initial state obtained by applying local unitary transformation will yield the same result.

This last result clearly illustrates the fact that for given values of $\alpha^{2}$ the inseparability of the input state can be broadcasted by performing local operations. To appreciate more clearly this result we turn our attention to the copying of a separable state of the form

$$
\hat{\boldsymbol{\rho}}_{a_{I} a_{I I}}^{(i n)}=\sum_{i} w_{i} \hat{\boldsymbol{\rho}}_{a_{I, i}}^{(i n)} \otimes \hat{\boldsymbol{\rho}}_{a_{I I, i}}^{(i n)} .
$$

In this case it is easily seen that the output of our procedure is of the form

$$
\hat{\rho}_{a_{I} b_{I} a_{I I} b_{I I}}^{(\text {out })}=\sum_{i} u_{i} \hat{\rho}_{a_{I, i} b_{I, i}}^{(\text {out })} \otimes \hat{\rho}_{a_{I I, i}}^{\left(\text {out } b_{I I, i}\right.},
$$

from which it follows that in this case the output $\hat{\rho}_{a_{I} b_{I I}}^{(\text {out })}$ is always separable, i.e.,

$$
\hat{\rho}_{a_{I} b_{I I}}^{(\text {out })}=\sum_{i} v_{i} \hat{\rho}_{a_{I, i}}^{(\text {out })} \otimes \hat{\rho}_{b_{I I, i}}^{(\text {out })} .
$$

This illustrates the fact that the inseparability cannot be produced by two distant parties operating locally and who can communicate only classically. This result is not only related to our procedure, but is easily seen to be valid for general local operations and classical communications.

\section{CONCLUSION}

In conclusion, using a simple set of local operations that can be expressed in terms of quantum state copying [4] we have shown that inseparability of quantum states can be locally copied with the help of local quantum copiers. We will investigate elsewhere how close the distilled copied states $\hat{\widetilde{\boldsymbol{\rho}}}_{a_{I} b_{I I}}^{(\text {out })}$ and $\hat{\widetilde{\boldsymbol{\rho}}}_{b_{I} a_{I I}}^{(\text {out })}$ are to the distilled input state $\hat{\widetilde{\boldsymbol{\rho}}}_{a_{I} a_{I I}^{(i n)}}$ and in particular whether the efficiency of the quantum copying can be improved when we do not average over all possible output states of the quantum copier but perform measurements on the quantum copier (conditional output states). This will give us a qualitative measure of how well a pure quantum entanglement can be broadcasted. More importantly, we would like to generalize our procedure such that any amount of initial entanglement, no matter how small, can be split into two even less entangled states. We now know that an equivalent of such a general procedure exists for purification procedures [11]. This, when found, would give us operational means of quantifying the amount of entanglement [6].

\section{ACKNOWLEDGMENTS}

This work was supported by the United Kingdom Engineering and Physical Sciences Research Council, the grant agency VEGA of the Slovak Academy of Sciences (under Project No. 2/1152/96), the National Science Foundation under Grant No. INT 9221716, the European Union, the Alexander von Humboldt Foundation, and the Knight Trust.

\section{APPENDIX}

In this paper we have utilized one nontrivial quantumcopier transformation (13) with the help of which broadcasting of entanglement via local copying can be performed. Here we present a scheme by means of which one can, in principle, determine a class of local quantum-copier transformations such that local outputs of quantum copiers are described by separable density operators $\hat{\rho}_{a_{I} b_{I}}^{(\text {out })}$ and $\hat{\rho}_{a_{I I} b_{I I}}^{(\text {out })}$ while the nonlocal states $\hat{\rho}_{a_{I} b_{I I}}^{(o u t)}$ and $\hat{\rho}_{a_{I I} b_{I}}^{\text {out })}$ are inseparable.

The most general quantum-copier transformation for a single spin $1 / 2$ has the form

$$
\begin{gathered}
|0\rangle_{a}|Q\rangle_{x} \rightarrow \sum_{i=1}^{4}\left|R_{i}\right\rangle_{a b}\left|X_{i}\right\rangle_{x}, \\
|1\rangle_{a}|Q\rangle_{x} \rightarrow \sum_{i=1}^{4}\left|R_{i}\right\rangle_{a b}\left|Y_{i}\right\rangle_{x},
\end{gathered}
$$

where $\left|R_{i}\right\rangle_{a b}(i=1, \ldots, 4)$ are four basis vectors in the fourdimensional Hilbert space of the output modes $a$ and $b$. These vectors are defined as $\left|R_{1}\right\rangle=|00\rangle,\left|R_{2}\right\rangle=|01\rangle$, $\left|R_{3}\right\rangle=|10\rangle$, and $\left|R_{4}\right\rangle=|11\rangle$. The output states $\left|X_{i}\right\rangle_{x}$ and $\left|Y_{i}\right\rangle_{x}$ of the quantum copier in the basis of four orthonormal quantum-copier states $\left|Z_{i}\right\rangle_{x}$ read

$$
\begin{aligned}
& \left|X_{i}\right\rangle_{x}=\sum_{k=1}^{4} C_{k}^{(i)}\left|Z_{k}\right\rangle_{x}, \\
& \left|Y_{i}\right\rangle_{x}=\sum_{k=1}^{4} D_{k}^{(i)}\left|Z_{k}\right\rangle_{x} .
\end{aligned}
$$

The amplitudes $C_{k}^{(i)}$ and $D_{k}^{(i)}$ specify the action of the quantum copier under consideration. From the unitarity of the transformation (A1) three conditions on these amplitudes follow:

$$
\begin{aligned}
& \sum_{k=1}^{4}\left|C_{k}^{(i)}\right|^{2}=1, \\
& \sum_{k=1}^{4}\left|D_{k}^{(i)}\right|^{2}=1, \\
& \sum_{k=1}^{4} C_{k}^{(i)} D_{k}^{(i)}=1 .
\end{aligned}
$$


The further specification of the amplitudes $C_{k}^{(i)}$ and $D_{k}^{(i)}$ depends on the tasks that should be performed by the quantum copier under consideration. This means that we have to specify these amplitudes in terms of constraints imposed on the output of the copier. These constraints (which can take form of specific equalities or inequalities) then define domains of acceptable values of $C_{k}^{(i)}$ and $D_{k}^{(i)}$.

To be specific, let us assume that the entangled state (14) is going to be broadcasted by two identical local quantum copiers defined by Eq. (A1). In this case the density operator $\hat{\rho}_{a_{I} b_{I} a_{I I} b_{I I}}^{\text {out }}$ describing the four-particle output of the two copiers reads (in what follows we assume the amplitudes $C_{k}^{(i)}$ and $D_{k}^{(i)}$ to be real)

$$
\begin{aligned}
\hat{\rho}_{a_{I} b_{I} a_{I I} b_{I I}}^{(o u t)} & \sum_{i_{I}, i_{I I}, j_{I}, j_{I I}} \sum_{k_{I}, k_{I I}} \omega_{k_{I} k_{I I}}^{\left(i_{I} i_{I I}\right)} \omega_{k_{I} k_{I I}}^{\left(j_{I I} j_{I I}\right)} \\
& \times\left|R_{i_{I}}\right\rangle_{a_{I} b_{I}}\left\langle R_{j_{I}}|| R_{i_{I I}}\right\rangle_{a_{I I} b_{I I}}\left\langle R_{j_{I I}}\right|,
\end{aligned}
$$

where

$$
\omega_{k l}^{(i j)}=\alpha C_{k}^{(i)} C_{l}^{(j)}+\beta D_{k}^{(i)} D_{l}^{(j)}
$$

The local output of the quantum copier $X_{I}$ is now described by the density operator $\hat{\rho}_{a_{I} b_{I}}^{(o u t)}$, which can be expressed as

$$
\hat{\rho}_{a_{I} b_{I}}^{(\text {out })}=\sum_{i_{I}, j_{I}} \Xi^{\left(i_{I} j_{I}\right)}\left|R_{i_{I}}\right\rangle_{a_{I} b_{I}}\left\langle R_{j_{I}}\right|
$$

where the matrix elements $\Xi^{\left(i_{I} j_{I}\right)}$ of this density operator in the basis $\left|R_{i_{I}}\right\rangle_{a_{I} b_{I}}$ read

$$
\Xi^{\left(i_{I} j_{I}\right)}=\sum_{k} \alpha^{2} C_{k}^{\left(i_{I}\right)} C_{k}^{\left(j_{I}\right)}+\beta^{2} D_{k}^{\left(i_{I}\right)} D_{k}^{\left(j_{I}\right)}
$$

In our discussion of broadcasting of entanglement we have assumed that local outputs of quantum copiers $X_{I}$ and $X_{I I}$ are separable. This implies restrictions on the density operator $\hat{\rho}_{a_{I} b_{I}}^{(o u t)}$, i.e., the four eigenvalues of the partially transposed operator $\left[\hat{\rho}_{a_{I} b_{I}}^{(\text {out })}\right]^{T_{2}}$ have to be positive $[7,8]$. So these are four additional constraints on the amplitudes $C_{k}^{(i)}$ and $D_{k}^{(i)}$ [the first three constraints are given by Eq. (A2)]. Further constraints are to be obtained from the assumption that the density operator $\hat{\rho}_{a_{I} b_{I I}}^{(o u t)}$ is inseparable. The explicit expression for this density operator can be expressed in the form

$$
\hat{\rho}_{a_{I} b_{I I}(o u t)}=\sum_{i_{I}, j_{I I}} \Omega^{\left(i_{I} j_{I I}\right)}\left|R_{i_{I}}\right\rangle_{a_{I} b_{I I}}\left\langle R_{j_{I I}}\right|
$$

where the diagonal matrix elements $\Omega^{\left(i_{I} j_{I I}\right)} \mathrm{read}$

$$
\begin{aligned}
\Omega^{(1,1)}= & \sum_{k, l}\left[\omega_{k l}^{(1,1)} \omega_{k l}^{(1,1)}+\omega_{k l}^{(2,1)} \omega_{k l}^{(2,1)}\right. \\
& \left.+\omega_{k l}^{(1,3)} \omega_{k l}^{(1,3)}+\omega_{k l}^{(2,3)} \omega_{k l}^{(2,3)}\right],
\end{aligned}
$$

$$
\begin{aligned}
\Omega^{(2,2)}= & \sum_{k, l}\left[\omega_{k l}^{(1,2)} \omega_{k l}^{(1,2)}+\omega_{k l}^{(2,2)} \omega_{k l}^{(2,2)}\right. \\
& \left.+\omega_{k l}^{(1,4)} \omega_{k l}^{(1,4)}+\omega_{k l}^{(2,4)} \omega_{k l}^{(2,4)}\right], \\
\Omega^{(3,3)}= & \sum_{k, l}\left[\omega_{k l}^{(3,1)} \omega_{k l}^{(3,1)}+\omega_{k l}^{(4,1)} \omega_{k l}^{(4,1)}\right. \\
& \left.+\omega_{k l}^{(3,3)} \omega_{k l}^{(3,3)}+\omega_{k l}^{(4,3)} \omega_{k l}^{(4,3)}\right], \\
\Omega^{(4,4)}= & \sum_{k, l}\left[\omega_{k l}^{(4,2)} \omega_{k l}^{(4,2)}+\omega_{k l}^{(3,2)} \omega_{k l}^{(3,2)}\right. \\
& \left.+\omega_{k l}^{(3,4)} \omega_{k l}^{(3,4)}+\omega_{k l}^{(4,4)} \omega_{k l}^{(4,4)}\right] .
\end{aligned}
$$

For the off-diagonal matrix elements we find

$$
\begin{aligned}
\Omega^{(2,1)}= & \sum_{k l}\left[\omega_{k l}^{(2,2)} \omega_{k l}^{(2,1)}+\omega_{k l}^{(1,2)} \omega_{k l}^{(1,1)}\right. \\
& \left.+\omega_{k l}^{(1,4)} \omega_{k l}^{(1,3)}+\omega_{k l}^{(2,4)} \omega_{k l}^{(2,3)}\right]=\Omega^{(1,2)},
\end{aligned}
$$

$$
\begin{aligned}
\Omega^{(3,1)}= & \sum_{k l}\left[\omega_{k l}^{(3,1)} \omega_{k l}^{(1,1)}+\omega_{k l}^{(4,1)} \omega_{k l}^{(2,1)}\right. \\
& \left.+\omega_{k l}^{(3,3)} \omega_{k l}^{1,3}+\omega_{k l}^{(4,3)} \omega_{k l}^{(2,3)}\right]=\Omega^{(1,3)},
\end{aligned}
$$

$$
\begin{aligned}
\Omega^{(4,1)}= & \sum_{k l}\left[\omega_{k l}^{(3,2)} \omega_{k l}^{(1,1)}+\omega_{k l}^{(4,2)} \omega_{k l}^{(2,1)}\right. \\
& \left.+\omega_{k l}^{(3,4)} \omega_{k l}^{(1,3)}+\omega_{k l}^{(4,4)} \omega_{k l}^{(2,3)}\right]=\Omega^{(1,4)},
\end{aligned}
$$

$$
\begin{aligned}
\Omega^{(3,2)}= & \sum_{k l}\left[\omega_{k l}^{(1,2)} \omega_{k l}^{(3,1)}+\omega_{k l}^{(2,2)} \omega_{k l}^{(4,1)}\right. \\
& \left.+\omega_{k l}^{(1,4)} \omega_{k l}^{(3,3)}+\omega_{k l}^{(2,4)} \omega_{k l}^{(4,3)}\right]=\Omega^{(2,3)}, \\
\Omega^{(4,2)}= & \sum_{k l}\left[\omega_{k l}^{(1,2)} \omega_{k l}^{(3,1)}+\omega_{k l}^{(2,2)} \omega_{k l}^{(4,2)}\right. \\
& \left.+\omega_{k l}^{(1,4)} \omega_{k l}^{(3,4)}+\omega_{k l}^{(2,4)} \omega_{k l}^{(4,4)}\right]=\Omega^{(2,4)}, \\
\Omega^{(3,4)}= & \sum_{k l}\left[\omega_{k l}^{(3,1)} \omega_{k l}^{(3,2)}+\omega_{k l}^{(4,1)} \omega_{k l}^{(4,2)}\right. \\
& \left.+\omega_{k l}^{(3,3)} \omega_{k l}^{(3,4)}+\omega_{k l}^{(4,3)} \omega_{k l}^{(4,4)}\right]=\Omega^{(4,3)} .
\end{aligned}
$$

If the density operator is supposed to be inseparable then at least one of the eigenvalues of the partially transposed operator $\hat{\rho}_{a_{I} b_{I I}}^{(\text {out })}$ has to be negative. This represents another con- 
dition that specifies the amplitudes $C_{k}^{(i)}$ and $D_{k}^{(i)}$.

We have to note that the conditions we have derived result in a set of nonlinear equations that are very difficult to solve explicitly. Moreover, these equations do not specify the amplitudes uniquely, so more constraints have to be found. Obviously, it will then become more difficult to check whether there exist some amplitudes $C_{k}^{(i)}$ and $D_{k}^{(i)}$ that fulfill these constraints.
[1] A. Ekert, Phys. Rev. Lett. 67, 661 (1991); C.H. Bennett, G. Brassard, and N.D. Mermin, ibid. 68, 557 (1992).

[2] W.K. Wootters and W.H. Zurek, Nature 299, 802 (1982).

[3] H. Barnum, C.M. Caves, C.A. Fuchs, R. Jozsa, and B. Schumacher, Phys. Rev. Lett. 76, 2818 (1996).

[4] V. Bužek and M. Hillery, Phys. Rev. A 54, 1844 (1996); see also M. Hillery and V. Bužek (unpublished).

[5] C.H. Bennett, H.J. Bernstein, S. Popescu, and B. Schumacher, Phys. Rev. A 53, 2046 (1996); D. Deutsch, A. Ekert, R. Josza, C. Macchiavello, S. Popescu, and A. Sanpera, Phys. Rev. Lett.
77, 2818 (1996).

[6] V. Vedral, M.B. Plenio, M.A. Rippin, and P.L. Knight, Phys. Rev. Lett. 78, 2274 (1997).

[7] A. Peres, Phys. Rev. Lett. 77, 1413 (1996).

[8] M. Horodecki, P. Horodecki, and R. Horodecki, Phys. Lett. A 223, 1 (1996).

[9] D. Bures, Trans. Am. Math. Soc. 135, 199 (1969); see also A. Uhlmann, Rep. Math. Phys. 9, 273 (1976); 24, 229 (1986).

[10] C.A. Fuchs and A. Peres, Phys. Rev. A 53, 2038 (1996).

[11] M. Horodecki, P. Horodecki, and R. Horodecki, Phys. Rev. Lett. 78, 574 (1997). 